\title{
Pion scattering with the LArIAT experiment
}

\author{
Justin Hugon* \\ Louisiana State University \\ E-mail: jhugonelsu.edu
}

This paper presents studies of pion scattering on liquid argon (LAr) with the LArIAT experiment. Pion scattering cross-sections on LAr are an important input to models of neutrino scattering used by current and future LAr neutrino experiments, such as MicroBooNE, SBND, and DUNE. LArIAT is a small LAr time projection chamber (LArTPC) in an instrumented testbeam at Fermilab. The precise calorimetry and tracking of LArTPC technology enable reconstruction of an interacting pion's energy as well as identification of secondary particles produced in the interaction. This makes possible measurement of the total pion-argon cross-section and exclusive interaction channels such as pion absorption, charge exchange, and scattering.

The 19th International Workshop on Neutrinos from Accelerators-NUFACT2017

25-30 September, 2017

Uppsala University, Uppsala, Sweden

* Speaker.

${ }^{\dagger}$ On behalf of the LArIAT Collaboration. 


\section{Introduction}

The United States neutrino program has decided to use LArTPC technology for the future of its experiments in both long-baseline neutrino oscillations with DUNE [1] and short-baseline neutrino oscillations with ICARUS [2, 3], MicroBooNE [4], and SBND [3]. All of these experiments require accurate estimates of oscillated neutrino flavor and energy, which are reconstructed from charged particles and electromagnetic showers, byproducts of the neutrino interactions in LAr. These charged particles and electromagnetic showers must also be reconstructed accurately in the LArTPC. In addition, to accurately estimate the neutrino flavor and energy, charged pion interactions that occur before leaving the neutrino interaction nucleus must also be taken into account. DUNE will also search for physics beyond the standard model of particle physics, including searching for proton decay to a $\mathrm{K}^{+}$and neutron-anti-neutron oscillations, which involve anti-nucleon annihilation at rest. The Liquid Argon in a Testbeam (LArIAT) experiment [5] contributes to all of these goals by improving our understanding of particle interactions in LAr and improving LArTPC event reconstruction.

\section{LArIAT Detector and Beamline}

The LArIAT time projection chamber (TPC) and cryostat have been re-purposed from the ArgoNEUT [6] experiment. The TPC and its dimensions are shown in Figure 1a. The TPC contains $170 \mathrm{~L}$ (0.25 tonnes) of LAr.

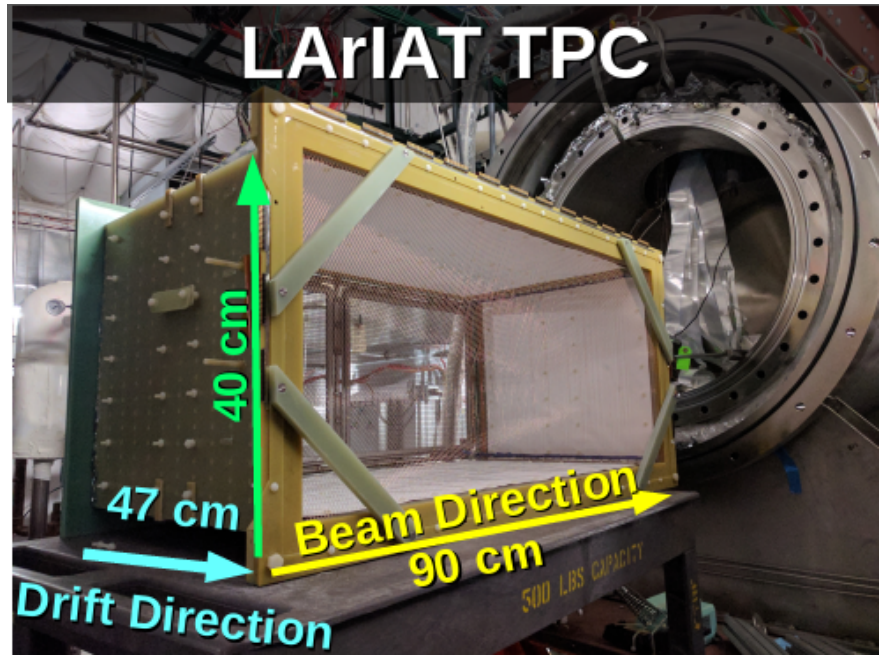

(a)

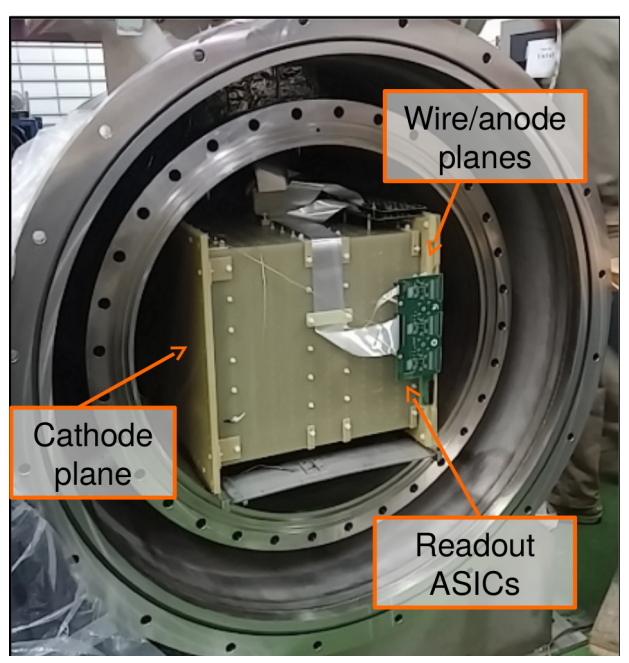

(b)

Figure 1: Pictures of the LArIAT TPC outside of the cryostat showing the dimensions (a) and of the TPC in the cryostat showing the cold front-end amplifiers (b). The TPC holds $170 \mathrm{~L}$ ( 0.25 tonnes) of LAr.

New wire planes have been installed on the TPC, as well as new cold front-end amplifiers from the MicroBooNE experiment [4, 7]; the cryostat and TPC have also been modified to include 
scintillation light detectors. Data has been taken with three different wire pitches: $3 \mathrm{~mm}, 4 \mathrm{~mm}$, and $5 \mathrm{~mm}$. The $4 \mathrm{~mm}$ data is used for hadronic cross-sections, scintillation light studies with PMTs and SiPMs, and general $\mathrm{R} \& \mathrm{D}$. The $3 \mathrm{~mm}$ and $5 \mathrm{~mm}$ datasets allow LArIAT to compare particle identification performance between different wire pitch configurations, and include a mesh cathode and the new ARAPUCA [8] light detection system. The new front-end amplifiers are located on the TPC immersed in LAr, as shown in Figure 1b. The low-temperature at this location and short distance to the readout wires reduce the amount of readout noise. The output signals of the frontend amplifiers are then brought out of the cryostat and fed into commercial digitizers.

The LArIAT experiment is located in the Fermilab Test-Beam Facility (FTBF). Fermilab's Main Injector sends $120 \mathrm{GeV}$ protons to the FTBF. The FTBF then creates a tunable secondary beam of $8 \mathrm{GeV}$ to $80 \mathrm{GeV}$ pions that is sent to the LArIAT experimental hall. This beam then hits a copper target creating the low energy tertiary beam that is directed at LArIAT. Figure 2 shows a diagram of the tertiary beamline. The dipole magnet and collimators are used to select a certain particle momentum range to go into the TPC, and the multi-wire proportional chambers precisely measure the momentum, position, and direction of the particle. The other detectors, such as the time-of-flight (TOF) counters, help identify the type of particle entering the TPC.

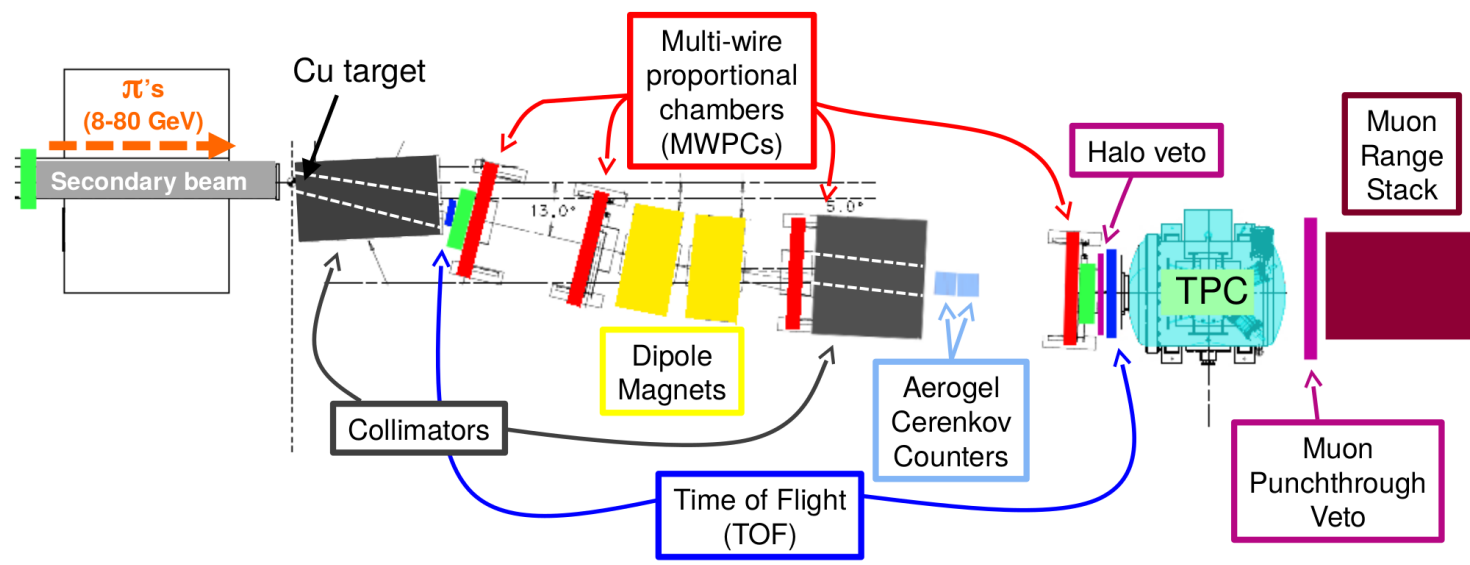

Figure 2: A diagram of the LArIAT tertiary beamline showing the location of the LArIAT TPC.

\section{Pion Cross-section}

The $\pi^{-}$total cross-section on Ar begins with a beamline event selection. Candidate beamline tracks are required to have a mass, calculated from the dipole magnetic field and hits in the wirechambers and TOF counters, consistent with a pion. The beamline tracks are then extrapolated to the TPC and matched with TPC tracks. Their position at the upstream face of the TPC must be within $5 \mathrm{~cm}$ in the horizontal and vertical directions and their 3D angles must be within $10^{\circ}$ of each other. Cuts on additional TPC tracks are used to reduce the electron background.

A novel use of the thin slice formula is used to to estimate the cross-section. The formula usually assumes the situation shown in Figure $3 \mathrm{a}$ and is

$$
\sigma(K E)=\frac{1}{n z} \frac{N_{\text {interacting }}(K E)}{N_{\text {incident }}(K E)},
$$


where $n$ is the number density of Ar nuclei per unit volume, $\mathrm{z}$ is the thickness of the thin slice $N_{\text {incident }}(K E)$ is the number of events incident on the target with a given kinetic energy, and $N_{\text {interacting }}(K E)$ is the number of events that interact with a given kinetic energy.

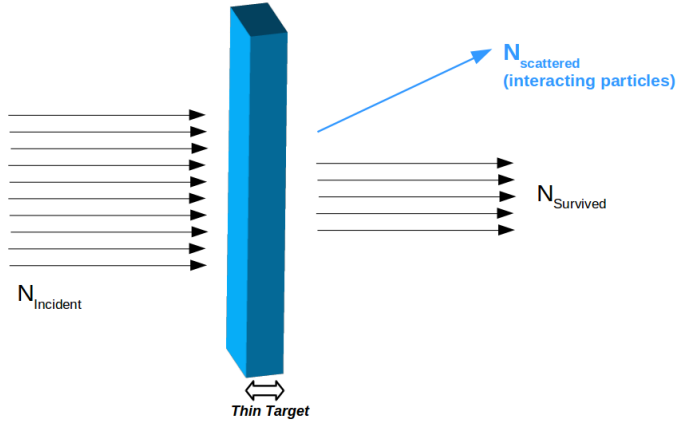

(a)

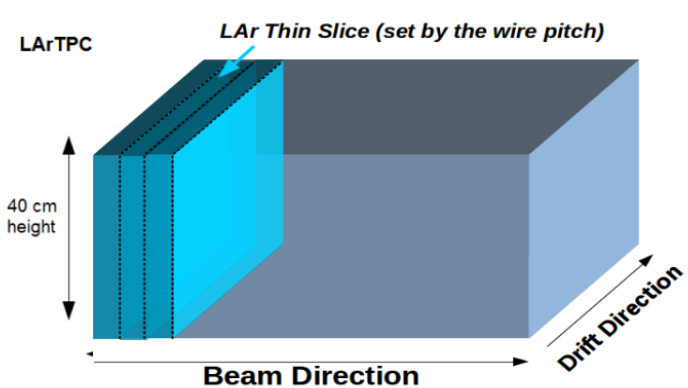

(b)

Figure 3: A diagram illustrating a thin slice target with incident and interacting particles (a) and a LArTPC divided into thin slices by the pitch of the readout wires (b).

Our variation on the method is that each wire with a signal from the track (that has a hit) is used as a separate thin slice, greatly multiplying the number of measurements that go into the cross-section estimate. It is as if the detector is many thin slices as shown in Figure $3 b$. The pion kinetic energy is estimated at each hit, so that each hit along a track fills a $N_{\text {incident }}(K E)$ histogram, while the final hit along a track, where it interacts, fills a $N_{\text {interacting }}(K E)$ histogram.

The initial kinetic energy of the pion at its first track hit is estimated as

$$
K E_{0}=\sqrt{p^{2}+m_{\pi}^{2}}-m_{\pi}-E_{\text {Flat }}
$$

where $p$ is the momentum measured by the beamline, $m_{\pi}$ is the mass of a pion, and $E_{\text {Flat }}$ is the amount of energy lost before the pion reaches the active region of the TPC. $E_{\text {Flat }}$ is estimated from simulation and is approximately flat with respect to KE. The KE of each following hit is estimated as the KE of the previous hit less the energy deposited in the previous hit, which is estimated from the charge deposited on the wire:

$$
K E_{\mathrm{i}}=K E_{\mathrm{i}-1}-E_{\text {Wire }}
$$

The incident and interacting data and simulation histograms for the pion sample are shown in Figure 4. Differences between data and simulation are under investigation. Mis-modeling of the composition of the incoming beam may be at fault.

The cross-section is shown in Figure 5. Note that the result has not been corrected for detector efficiency or energy resolution. The following systematic uncertainties have been taken into account: $d E / d x$ calibration, $3 \%$, energy loss prior to entering the TPC, $3.5 \%$, through going muon background contamination, $4 \%$, beamline momentum uncertainty, $3 \%$. 


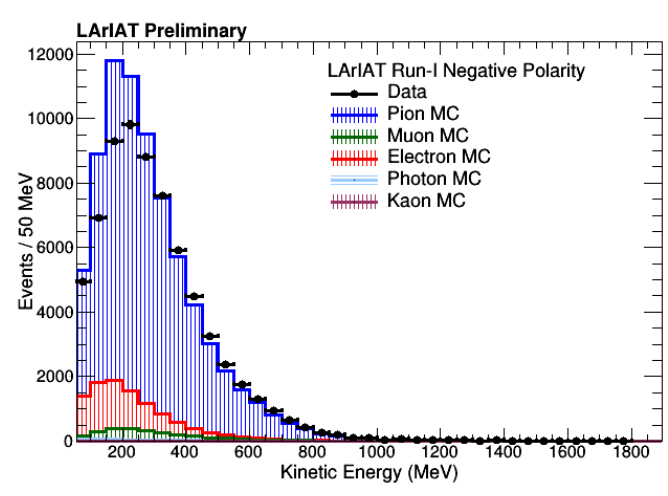

(a)

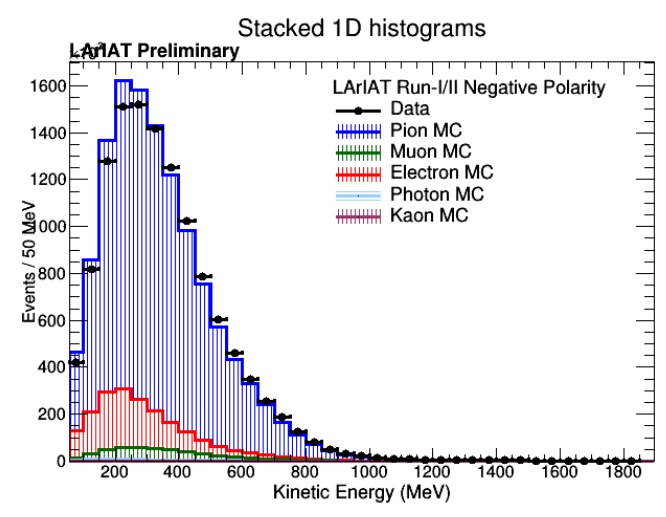

(b)

Figure 4: Data and simulation (labeled MC) of the interacting (a) and incident (b) histograms for the pion sample. The y-axis of (b) is multiplied by a factor of $10^{3}$.

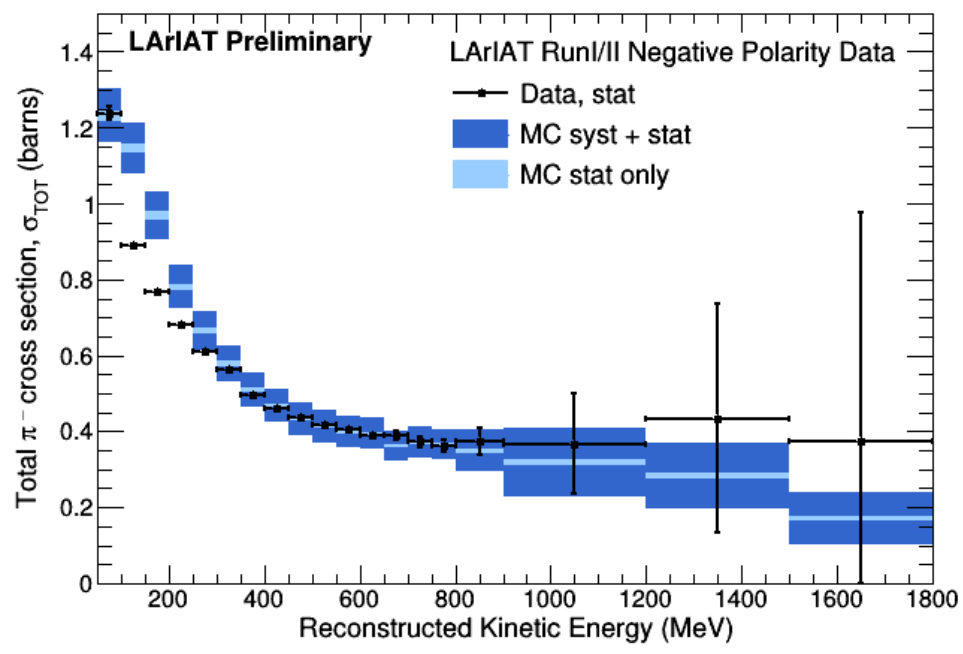

Figure 5: The measured total $\pi^{-}$cross-section on Ar.

\section{Toward Exclusive Pion Channels}

In addition to the total $\pi^{-}$cross-section, many other cross-section studies are in progress for LArIAT. One of these is measuring the $\pi^{+}$absorption plus charge exchange reaction, $\pi^{+}+\mathrm{Ar} \rightarrow$ $0 \pi^{ \pm}+X$. The selection for this channel is similar to the one for the total cross-section, but requires the absence of charged pions in the final state. This requires a particle identification algorithm to separate charged pion tracks from proton tracks. The author has been working on an algorithm using a simulation-derived likelihood for each track hit. In the simulation, for each hit on a pion or proton track, 2D histograms of $\mathrm{dE} / \mathrm{dx}$ versus residual range are filled. Residual range is the distance from a hit to the end of a track along the trajectory of the track. These histograms are 
then normalized and used as likelihoods and are shown in Figures 6a and 6b. As you can see, the protons are more ionizing than the pions. The likelihood for a track to be pion or proton can then be found as the product of the likelihood of each hit over all of the hits in a track. The ratio of the pion and proton likelihoods (or difference in the log-likelihoods) for a track can then be used to discriminate between pions and protons. Figure $6 \mathrm{c}$ shows that the log-likelihood separates the pions and protons well. These simulated events are a statistically independent sample from the one used to create the likelihoods of Figures $6 a$ and $6 b$.

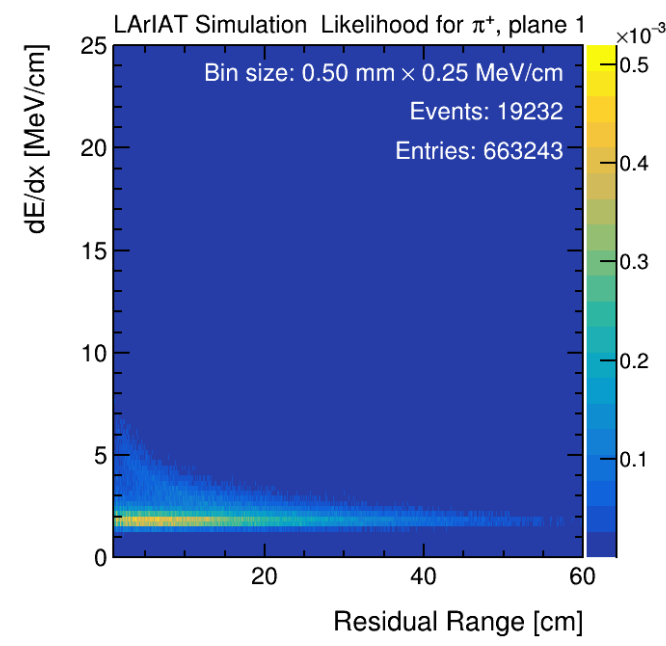

(a)

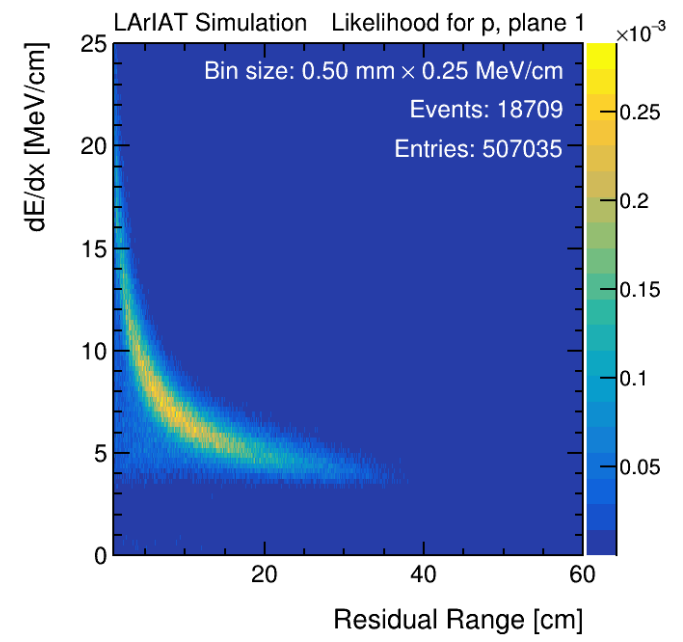

(b)

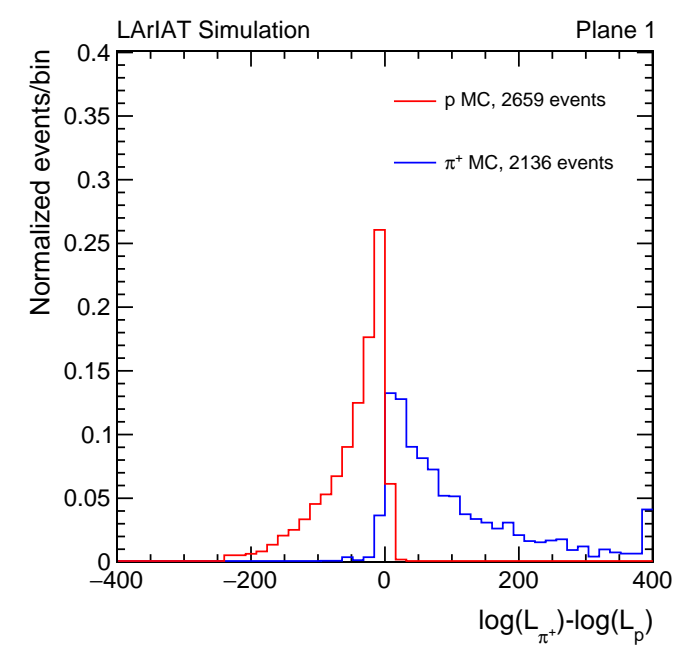

(c)

Figure 6: Likelihood histograms of $d E / d x$ versus residual range for pions (a) and protons (b). The difference in the log-likelihoods for simulated pion and proton samples (c). 


\section{Conclusions}

The LArIAT experiment has made a preliminary measurement of the $\pi^{-}$total cross-section. Many other cross-section measurements on LAr are in progress, including ones not discussed here with kaons and anti-protons. These measurements will all help improve understanding of LArTPC detectors to further the DUNE and short-baseline neutrino scientific programs.

\section{References}

[1] DUNE Collaboration, Long-Baseline Neutrino Facility (LBNF) and Deep Underground Neutrino Experiment (DUNE) Conceptual Design Report, Volume 1: The LBNF and DUNE Projects (2016). arXiv: 1601.05471

[2] ICARUS, Design, construction and tests of the ICARUS T600 detector. Nucl. Instrum. Meth. A 527, 329 (2004). doi:10.1016/j.nima.2004.02.044

[3] MicroBooNE, LAr1-ND, and ICARUS-WA104 Collaborations, A Proposal for a Three Detector Short-Baseline Neutrino Oscillation Program in the Fermilab Booster Neutrino Beam (2015). arXiv: 1503.01520

[4] MicroBooNE Collaboration, Design and Construction of the MicroBooNE Detector. JINST 12, P02017 (2017). doi:10.1088/1748-0221/12/02/P02017. arXiv:1612.05824

[5] LArIAT Collaboration, LArIAT: Liquid Argon In A Testbeam (2014). arXiv:1406.5560

[6] ArgoNeuT Collaboration, The ArgoNeuT Detector in the NuMI Low-Energy Beam Line at Fermilab (2014). arXiv:1205.6747

[7] G. De Geronimo et al., Front-end ASIC for a Liquid Argon TPC. IEEE Trans. Nucl. Sci. 58, 1376 (2011). doi:10.1109/TNS.2011.2127487

[8] A.A. Machado, E. Segreto, ARAPUCA a new device for liquid argon scintillation light detection. JINST 11, C02004 (2016). doi:10.1088/1748-0221/11/02/C02004 